\title{
Real-Time Controlled Multi-objective Scheduling Through ANNs and Fuzzy Inference Systems: The Case of DRC Manufacturing
}

\author{
Ozlem Uzun Araz \\ Dokuz Eylul University Industrial Engineering Department, 35100, Izmir, Turkey \\ ozlem.uzun@deu.edu.tr
}

\begin{abstract}
In this paper, we developed an integrated multi-objective real-time controlled scheduling methodology for Dual Resource Constrained systems. The proposed methodology integrates simulation, neural network and fuzzy inference system approaches to obtain a schedule considering both state of the system and objectives. By means of a case study, we have demonstrated that the proposed methodology can be an effective tool for dynamic scheduling of DRC systems.
\end{abstract}

Keywords: Dynamic Scheduling, Fuzzy Inference Systems, Artificial Neural Networks, Dual Resource Constrained Manufacturing System.

\section{Introduction}

Scheduling decisions are increasingly seen as strategic functions for manufacturing firms. Because of its increasing importance, many researchers have paid attention to develop numerous scheduling approaches for machine-constrained manufacturing systems. However, until recent years, the scheduling of Dual-Resource Constrained (DRC) manufacturing systems has not received much attention in the literature. Scheduling of these systems is more complicated than the others. Most of the DRC offline scheduling approaches developed in the literature use dispatching rules [1, 2, 3, 4, 5, etc.].

A drawback, however, of using dispatching rules is that their performances are dependent on the state of the system, but there is no rule that is the best performer for all the possible state of the system [6]. Therefore, for flexible and dynamic scheduling (DS) decisions, an effective tool is required to help the decision maker in selecting the best rule for each particular state of the system. Many researchers have proposed simulation and dispatching rule based dynamic scheduling approaches for machine constrained manufacturing systems $[7,8,9$, etc]. Due to the time consuming nature of simulation, some researchers have developed the artificial intelligence techniques for effective solutions of dynamic scheduling problems [10,11, etc.].

To the best of our knowledge, the applicability and effectiveness of the artificial neural networks (ANN) based dynamic scheduling methods have not yet been fully explored on complex DRC manufacturing systems. In this paper, a novel real-time controlled multi-objective DRC scheduler is proposed to dynamically select dispatching rules. The proposed methodology, tested in a hypothetical manufacturing system, proves to be an effective method in a dynamic DRC manufacturing system. 


\section{The Multi-objective DRC Scheduler}

The proposed scheduler (see Fig. 1) integrates several tools, namely; a simulation model, a backpropagation neural network (BPNN) and a fuzzy inference system (FIS), to dynamically select dispatching rules. A simulation experiment is conducted to collect predefined performance measures corresponding to the current dispatching rule set and the system states. BPNN is used to obtain the performance measures for each shop configuration. A shop configuration considers various decision variables such as dispatching rule sets, due date tightness, arrival rates, etc. Then the system is real time controlled and its status is analyzed in order to detect pre-defined symptoms. When a symptom becomes active, BPNN generates performance measures for all possible shop configurations. In order to compare the system performance between all shop configurations, an aggregated performance measure for each configuration is provided using a fuzzy inference system. Finally, the best configuration is determined and used until a new symptom becomes active.

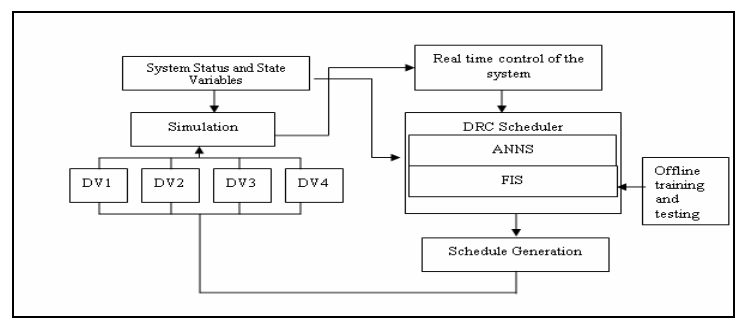

Fig. 1. The proposed real time scheduling mechanism. DV1: decision variables 1, etc

\section{An Application of DS for DRC Manufacturing System}

In order to investigate the potentials and effectiveness of the proposed methodology, the hypothetical DRC manufacturing system that consists of 24 departments is studied. There are 43 machines and 25 workers in the shop floor. Ten different parts can be processed by several flexible routing sequences. In this study, four decision variables are considered for scheduling of the system.

- Queue disciplines: First in first out (FIFO), shortest processing time, earliest due date, shortest remaining processing times, critical ratio, minimum slack time, critical ratio/ shortest processing time.

- When labor assignment rules: Centralized and decentralized rules are selected to determine when the worker should move to another station.

- Where labor assignment rules: The longest number in queue (LNQ), the longest waiting time in the queue and the work center with job which has shortest processing time and traveling time rules are selected to schedule of the worker.

- Alternative routing selection: Smallest number in queue (SNQ), shortest processing time at an operation, lowest average utilization first. 
Four performance measures are considered to evaluate the alternative scheduling policies: mean tardiness, number of tardy jobs, mean flow time and throughput rate. Mean queue time, WIP, average and maximum utilization of machines and operators, arrival rate, flow allowance factor and number of part types in the system are selected as system state variables.

The simulation model was developed by using ARENA 3.0 simulation software. The simulation length is determined 50000 minutes and 10 independent replications. Then, BPNNs model for each performance measures are developed using 500 randomly generated training and testing samples. The inputs to the BPNNs are decision rule set and current system state variables, while the output of the system is the considered performance measures. After training and testing the ANN models, FIS model is generated by using MATLAB 7.0 fuzzy logic toolbox. The FIS system consists of four inputs which represent the performance measures of the DRC system, one output which is the aggregated fuzzy rating and 81 rules. The alternative which has highest rating score is selected as new schedule.

In order to investigate the effectiveness of the approach, it is compared with fixed scheduling approach (denoted as [1111]) in which "when rule" is centralized, "where rule" is LNQ, part selection by machine is FIFO, machine selection by part is SNQ. The manufacturing system is simulated for 15.000 minutes with both fixed scheduling and the proposed dynamic scheduling approaches. During the simulation period, system state variables were changed randomly in order to ensure the dynamic nature of the system. The fixed scheduling approach is regulated by fixed decision rules for decision variables at the start of the simulation and this decision rule set is not changed during the scheduling period.
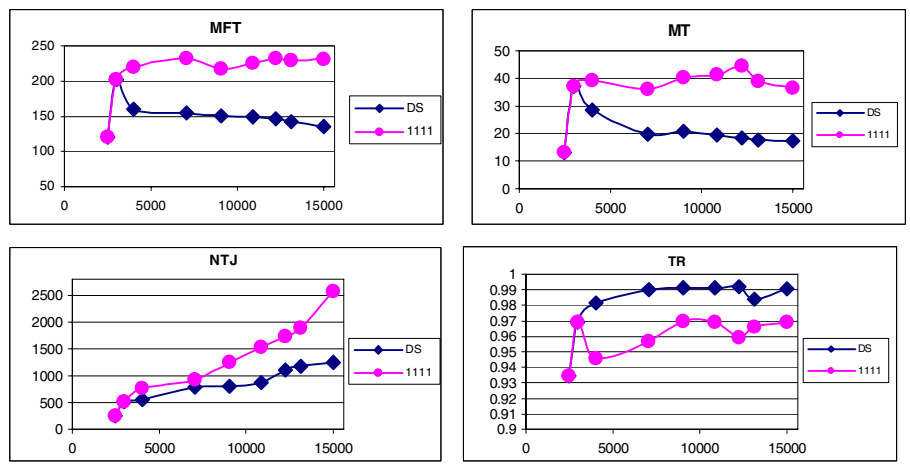

Fig. 2. Performance measures at each rescheduling point

During the application of dynamic scheduling approach, eight rescheduling points was detected. Performance measure values at each rescheduling point obtained from the fixed and dynamic scheduling approaches are illustrated in Fig. 2. As can be seen in figures, the proposed dynamic scheduling approach is more effective than the fixed scheduling approach in terms of all performance measures. It can easily be realized that when the value of a performance measure deteriorates, the proposed approach renews the schedule so that the overall performance of the system can be improved. It 
should also be noted that the performance of the proposed approach is compared with all alternative fixed rule sets. These results also prove that the proposed approach is superior to all fixed scheduling rules in terms of all performance measures.

\section{Conclusion}

In this paper, we presented a new multi-objective Dual Resource Constrained scheduler for dynamic scheduling. The proposed methodology integrates three tools namely; simulation, neural network and fuzzy inference system. In spite of the complexity of DRC manufacturing, the result of this study indicates that the proposed methodology is an effective method to find appropriate dispatching rules.

\section{References}

1. Nelson, R.T.: Labor and machine limited production systems. Management Science. 13 (1967) 648-671.

2. Treleven, M.: Applications and implementation. The timing of labor transfers in a dual resource-constrained systems: "push" vs. "pull" rules. Decision Sciences (1987) 18 73-88.

3. Bobrowski, P.M., Park, P.S.: An evaluation of labor assignment rules when workers are not perfectly interchangeable. Journal of Operation Management. 11(1993) 257-268.

4. Malhotra, M.K., Kher, H.V.: An evaluation of worker assignment policies in dual resource-constrained job shops with heterogeneous resources and worker transfer delays. International Journal of Production Research. 32 (1994) 1087-1103.

5. Bokhorst, J.A.C., Slomp, J., Gaalman, G.J.C.: On the who-rule in Dual Resource Constrained manufacturing systems. Int. J. Prod. Res. 42 (23) (2004) 5049-5074.

6. Priore, P., Fuente, D., Puente, J., Parreno, J. : A comparison of machine-learning algorithms for dynamic scheduling of flexible manufacturing systems. Engineering Applications of Artificial Intelligence. 19(3) (2006) 247-255.

7. Kim, C.O., Kim, Y.D. Simulation based real-time scheduling in a flexible manufacturing system. Journal of Manufacturing System. 13 (1994) 85-93.

8. Sabuncuoğlu, I., Kızılışık, O.B.: Reactive scheduling in a dynamic and stochastic FMS environment. International Journal of Production Research. 41(17) (2003) 4211-4231.

9. Chan, F.T.S., Chan, H.K., Lau, H.C.W., Ip, R.W.L.: Analysis of dynamic dispatching rules for a flexible manufacturing system. Journal of Material Processing Technology. 138 (2003) 325-331.

10. Min, H.S., Yih, Y., Kim, C.O.: A competitive neural network approach to multi-objective FMS scheduling. International Journal of Production Research. 36(7) (1998) 1749-1765.

11. Min, H.S., Yih, Y.: Selection of dispatching rules on multiple dispatching decision points in real-time scheduling of a semiconductor wafer fabrication system. International Journal of Production Research. 41(16) (2003) 3921-3941. 